\title{
Complex networks of interaction of genes located in the critical region of Down syndrome expressed in the normal human brain.
}

\author{
Dianora Fajardo ${ }^{*}$, Karla Vinasco ${ }^{2}$, Julio C Montoya ${ }^{3}$, Jose M Satizabal ${ }^{3}$, Adalberto Sanchez ${ }^{3}$, Felipe \\ García-Vallejo ${ }^{4}$ \\ ${ }^{1}$ Department of Biochemistry, Institución Universitaria Escuela Nacional del Deporte, Cali, Colombia \\ ${ }^{2}$ LABIOMOL, Universidad del Valle, Cali, Colombia \\ ${ }^{3}$ Department of Physiological Sciences, School of Basic Sciences, Universidad del Valle, Colombia \\ ${ }^{4}$ Scientific Director of the Laboratory of Molecular Biology and Pathogenesis, Department of Physiological Sciences, \\ School of Basic Sciences, Universidad del Valle, Cali, Colombia
}

\begin{abstract}
The quantification and analysis of the human transcriptome allows expanding the knowledge of the genomic functioning, especially in body's parts as complex and important as the human brain. In this way, in silico studies offer the possibility to extract and analyse information contained in databases, at the level of gene expression along the different brain structures. This study aimed to correlate the transcription levels of 38 genes located in the critical region of the chromosome 21 associated with Down syndrome with the cerebral localization and its intervention in the correct operation of different brain substructures. To carry out this, the expression profiles of these genes along 24 substructures of the brain cores and 18 of the Limbic lobe were done, from gene expression data of microarray experiments of DNA, available in the database of the Atlas of the brain of the "Allen Institute for Brain Sciences". It was determined a differential expression of these genes along the analysed structures, in addition to register higher levels of overall transcription in certain areas of the brain, which appear to be associated with different processes of learning and memory. The differential transcription was correlated with the cerebral localization and its potential functional role.
\end{abstract}

Keywords: Basal ganglia, Limbic lobe, DNA microarrays, Critical region of Down syndrome, PCP4, KCNJ6, DYRK1A.

Accepted on July 12, 2018

\section{Introduction}

The human brain has a wide variety of cells, each of those ones has a single morphophysiology, functionality and connectivity $[1,2]$. These properties are largely the result of unique combinations of expressed gene products, and its precise regulation is what keeps to a large cerebral homeostasis degree. This approach is useful for understanding the functional circuitry of the nervous system, and thus generates new knowledge about the relationship between genes, brain and behavior [3-5]. Therefore, the analysis of gene expression profiles provides huge information about brain connectivity and its relationship with the higher cognitive functions. As an example there is the influence of genetic factors in understanding the normal brain development and mental disorders [5-8]. Consequently, the cellular diversity of the brain requires a focus on understanding the functional genomics of the nervous system, leading to the incorporation of all these modalities in an overall analysis having the potential to improve the discovery and highlighting its importance in comparison with methods that analyse each mode separately. Data associations between these three approaches are relevant in the study of diseases, since gene expression in the brain plays a key role in the flow of information between brain networks and performing cognitive tasks $[3,5]$.

For that reason, the inherent importance of brain homeostasis and the complexity involved in the development and maintenance of the nervous system can result in a series of neuropathology that lead to changes in brain structure such as decreasing different types of motricity, cognitive impairment, among others [6]. Down Syndrome (DS), which is caused by the total or partial presence of three copies of chromosome 21, is becoming the most frequent aneuploidy leading to vary degrees of cognitive impairment [9-11]. On the other hand, studies of segmental trisomies have allowed to characterize an area within the chromosome called "Critical Region of the Down Syndrome" (DSCR), which is located at the distal end of the long arm of chromosome 21 (21q22.1-22.3), and which 
contains possible candidate genes whose imbalance of dose could induce marked cognitive deficit, like the other pathologies and traits associated [12-17].

However, the involvement of the DSCR as the sole cause of the symptoms of DS is still a matter of debate. Several studies suggest that this region plays a major role in the genetic interactions that would be related to the pathogenesis of DS [18-20]. In spite of this, the expression of the genes which are found within this region is not fully known in the brain. Therefore, their study in normal human brains could provide a better understanding of their participation in the regulation of all processes that must be performed for proper operation. Additionally, a comprehensive approach would make significant correlations between gene expression and regulation, the function of the nervous system and the resulting phenotype. This would be too informative for neurogenetic and study of brain diseases, especially in the neurological disorders associated with the DS.

Actually, few studies focus on the functional analysis correlation of gene expression in the brain. Moreover, the techniques that have been used, usually cover large regions of the brain creating a difficulty in interpreting data in the substructures, or generally made by a gene at a time, leaving patterns of expression of many genes uncharacterized. As a result, Atlas Allen Human Brain Project has adopted a global approach for understanding the structural and genetic architecture of the brain by generating gene expression profiles obtained from DNA microarrays from post-mortem human brains. In this context, it is possible to extract complete and detailed information on those levels of transcription in different brain structures that can be found in the database of free access of the Allen Brain Atlas [21]. This database contains anatomical and genomic information from human brains, which is supplemented with extra information and set of visualization tools and data mining. Microarray experiments include data of more than 62,000 probes, covering $93 \%$ of the 21,245 genes consigned, from which it is possible to obtain information on thousands transcriptional gene referenced with Entrez gene codes.

This study was designed to construct an in silico model of the expression profiles of 38 genes located in the critical region of DS. Data were based to correlate them with the functionality of a healthy human brain of a 55 y old male donor, besides, a network of expression and interaction of these genes with others that expression was built. The results allowed us to approach to a systemic model of expression that can be modified bioinformatically to extrapolate it to what happens in other diseases of the brain, providing a powerful tool for its understanding.

\section{Materials and Methods}

\section{Data collection}

The gene expression levels were calculated from the z-score values of 38 genes DSCR (Annex 1) in different substructures of the basal ganglia and the limbic lobe. These were obtained from the graphical display of the database of the human brain the Allen Institute for Brain Sciences. All procedures used for collecting data are reported extensively in the technical report "Allen Institute for Brain Sciences" [23].

In all cases, the data of each gene of available experiments using different probes was obtained. Standard values (z score) of the expression levels through 24 substructures of the brain nuclei and 18 from the Limbic Lobe (Annex 2) were recorded. These values were recorded in electronic sheets in Excel format for further analysis. Three values were taken at three different points of each substructure and the average $\mathrm{z}$ score was regarded as final data.

\section{Protein interaction network and cluster analysis}

A network of interaction of proteins encoded by 36 of the DSCR genes with other human proteins in different databases was built through a Cytoscape program version 3.1.1 [24]. Besides, a hierarchical clustering was performed based on the $\mathrm{z}$ score of these 36 genes in each cerebral substructure. The test conditions used were the plugin clusterMaker Algorithm maximum link by pairs and metric distance metric Pearson correlation.

\section{Statistical analysis}

For ranking each expression of genes in different brain substructures, a Principal Component Analysis (PCA) for both the limbic lobe and the brain nuclei was performed by reducing the $\mathrm{R}$ space of 38 collinear variables to 6 main components (R38 $>$ R6) in each area. This analysis was performed using IBM SPSS 20.0.0 program [25].

\section{Results}

\section{DSCR overall transcription of genes in the brain}

A differential expression of 38 genes DSCR along different brain substructures, specific and dependent on the associated substructure brain, was determined. In turn it was confirmed the existence of regulation of gene expression dependent on the physiology of each brain area (Figure 1). From the brain areas studied, the caudate nucleus, putamen and globus pallidus are substructures that had a higher level of expression by the majority of genes in the basal nuclei (Figure 1A). The most expressed areas in the limbic lobe were the central and the parahippocampal gyrus (Figure 1B).

The PCA allowed reducing to six components the 38 genes $D S C R$ in each structure. In brain, nuclei represented $79.89 \%$ of the variance while the limbic lobe $76.03 \%$. It was observed that the distribution of the $38 D S C R$ genes was differential in the two brain structures (Figure 2). In brain nuclei component 1 was the most complex, which includes 19 genes, in contrast to the limbic lobe where 19 of the 38 genes DSCR distributed between components 1 and 2 (Table 1 ). 

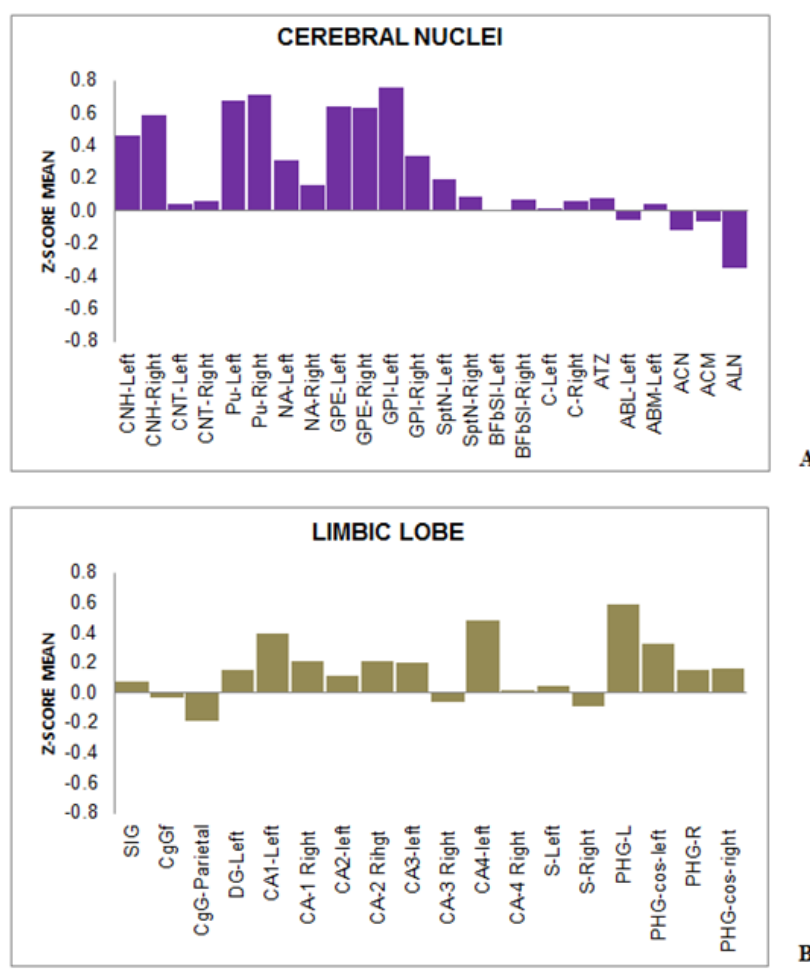

Figure 1. Comparison of the total average level of expression of all genes from in each brain substructure. A. Brain nuclei. B. Limbic lobe. The structures most expressed in the basal nuclei were caudate nucleus, putamen and globus pallidus, and for the limbic lobe were the central areas and the parahippocampal gyrus.

In addition, comparative analysis of graphics Biplot establishes two groups of genes having a statistically significant correlation for each structure. At Limbic lobe, the first association is composed by the IGSF5, C21orf88, TRAPPC10, TTC3, B3GALT5, DSCR3, KCNJ15 and C21orf24; and the second by the DSCR1, SIM2, RUNX1, ERG, DSCAM and $D S C R 4$ (Figure 2A) genes. While in brain nuclei, the stronger association included the BACE2, SIM2, ERG, CSTB, PTTG1IP, TMEM50B, TRAPPC10 genes (Figure 2B).

The PCA analysis showed that gene co-expression varies depending on the brain area. Both in brain nuclei and the limbic lobe, two groups presented where the genes that compose them are highly correlated, but all the associations that occur differ in both structures. Similarly, the cluster analysis showed the same pattern of co-expression (Figure 3), leaving also areas of high and low expression mentioned before, and the existence of an opposite expression of certain genes in these two areas.

\section{Protein interaction network}

The interaction network showed a master node that stands out within seven DSCR proteins interacting within them and with a variable number of others human proteins. The network showed that in the primary node DYRK1A gene product is found (Figure 4). It had one of the highest values of interactions within the network (Figure 5), and interacts directly with another six proteins DSCR: HMGN1, BRWD1, TTC3, RCAN1, ARE and DCSR3.
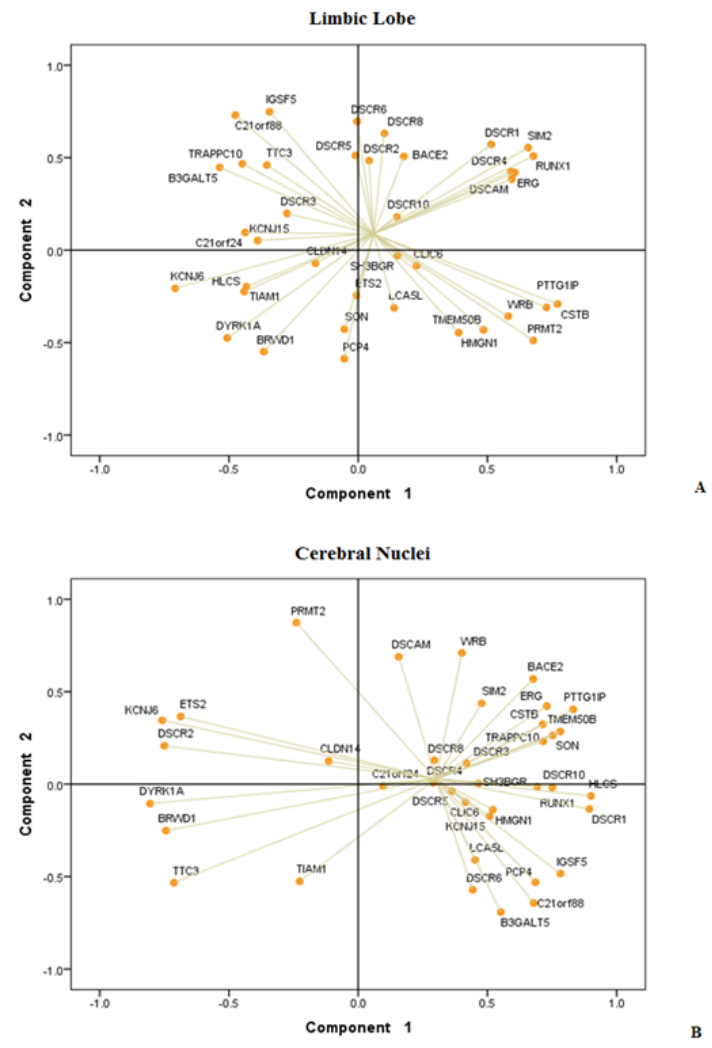

Figure 2. Biplot representation of the Cartesian plane that shows the distribution of genomic variables grouped because of the corresponding principal component analysis. A. Brain nuclei. B. Limbic lobe.

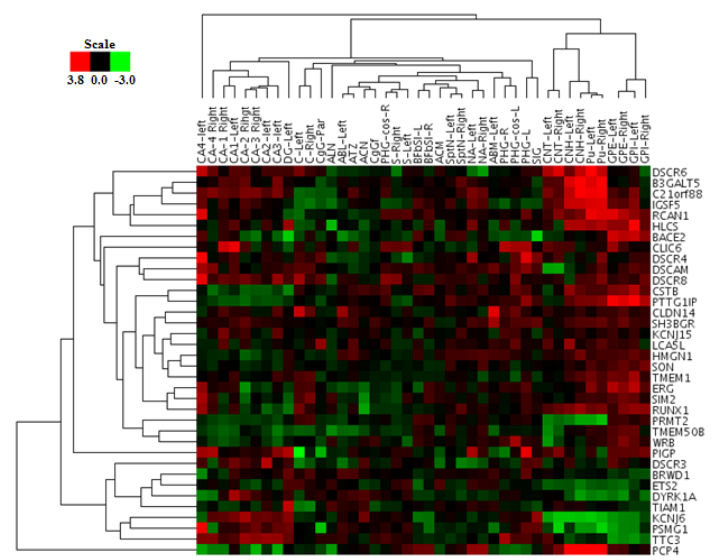

Figure 3. Cluster analysis of the expression of 38 genes located in the critical region SD along the various substructures of the limbic lobe and the brain nuclei. The structures with highest expression are represents in red, whilst the structures with less expression are represents in green.

In the same way, the top ten GO categories with highest statistical significance in terms of biological process were analysed. As shown in Table 2, most of the proteins studied in the interaction network regulate positively and/or negatively 
various biological and metabolic processes, mechanisms of apoptosis, modification of macromolecules, among others.

Table 1. Discrimination on the weight of each of the variables within six components.

\begin{tabular}{|c|c|c|c|c|c|c|c|}
\hline \multirow[t]{2}{*}{ Brain structure } & \multicolumn{7}{|l|}{ Component } \\
\hline & 1 & & 2 & 3 & 4 & 5 & 6 \\
\hline \multirow[t]{10}{*}{ Cerebral nuclei } & KCNJ15 & DSCR1 & DSCAM & DSCR4 & DSCR2 & CLIC6 & DSCR3 \\
\hline & $L C A 5 L$ & DSCR5 & ETS2 & DSCR6 & $B R W D 1$ & DYRK1A & TIAM1 \\
\hline & C21orf88 & DSCR10 & $W R B$ & DSCR8 & C21orf24 & & \\
\hline & $B 3 G A L T 5$ & BACE2 & PRMT2 & SIM2 & HMGN1 & & \\
\hline & TRAPPC10 & $E R G$ & & CLDN14 & KCNJ6 & & \\
\hline & IGF5 & RUNX1 & & TTC3 & & & \\
\hline & PCP4 & SH3BGR & & & & & \\
\hline & CSTB & SON & & & & & \\
\hline & TMEM50B & HLCS & & & & & \\
\hline & PTTG1IP & & & & & & \\
\hline \multirow[t]{10}{*}{ Limbic lobe } & DSCAM & & DSCR1 & DSCR10 & BRWD1 & KCNJ6 & DSCR3 \\
\hline & DSCR4 & & DSCR2 & CLDN14 & ETS2 & $S H 3 B G R$ & CLIC6 \\
\hline & $R U N X 1$ & & DSCR6 & KCNJ15 & SON & & DYRK1A \\
\hline & $E R G$ & & DSCR8 & C21orf24 & HLCS & & PCP4 \\
\hline & $W R B$ & & DSCR5 & LCA5L & HMGN1 & & \\
\hline & SIM2 & & BACE2 & B3GALT5 & TRAPPC10 & & \\
\hline & CSTB & & TTC3 & & TIAM1 & & \\
\hline & TMEM50B & & C21orf88 & & & & \\
\hline & PTTG1IP & & IGSF5 & & & & \\
\hline & PRMT2 & & & & & & \\
\hline
\end{tabular}

Table 2. Main GO categories of biological process established in the interaction network.

\begin{tabular}{|c|c|c|c|c|}
\hline GO-ID & Description & P-value & Cluster frequency & Total frequency \\
\hline 48523 & Negative regulation of cellular process & $5.35 \mathrm{E}-17$ & $179 / 702(25.4 \%)$ & $1840 / 14274(12.8 \%)$ \\
\hline 48519 & Negative regulation of biological process & $1.32 \mathrm{E}-15$ & $186 / 702(26.4 \%)$ & $2016 / 14274(14.1 \%)$ \\
\hline 6464 & Protein modification process & $1.49 \mathrm{E}-13$ & $148 / 702(21.0 \%)$ & $1527 / 14274(10.6 \%)$ \\
\hline 43412 & Macromolecule modification & $3.63 \mathrm{E}-13$ & $152 / 702(21.6 \%)$ & $1608 / 14274(11.2 \%)$ \\
\hline 10941 & Regulation of cell death & 1.13E-12 & $99 / 702(14.1 \%)$ & $868 / 14274(6.0 \%)$ \\
\hline 43067 & Regulation of programmed cell death & $1.46 \mathrm{E}-12$ & $98 / 702(13.9 \%)$ & $861 / 14274(6.0 \%)$ \\
\hline 9987 & Cellular process & $1.46 \mathrm{E}-12$ & $553 / 70278.7 \%$ & $9353 / 14274(65.5 \%)$ \\
\hline 42981 & Regulation of apoptosis & $1.73 \mathrm{E}-12$ & $97 / 702(13.8 \%)$ & $853 / 14274(5.9 \%)$ \\
\hline 48518 & Positive regulation of biological process & $3.67 \mathrm{E}-12$ & $186 / 70226.4 \%$ & $2207 / 14274(15.4 \%)$ \\
\hline 9893 & Positive regulation of metabolic process & $4.28 \mathrm{E}-12$ & $108 / 70215.3 \%$ & $1020 / 14274(7.1 \%)$ \\
\hline
\end{tabular}


Complex networks of interaction of genes located in the critical region of Down syndrome expressed in the normal human brain

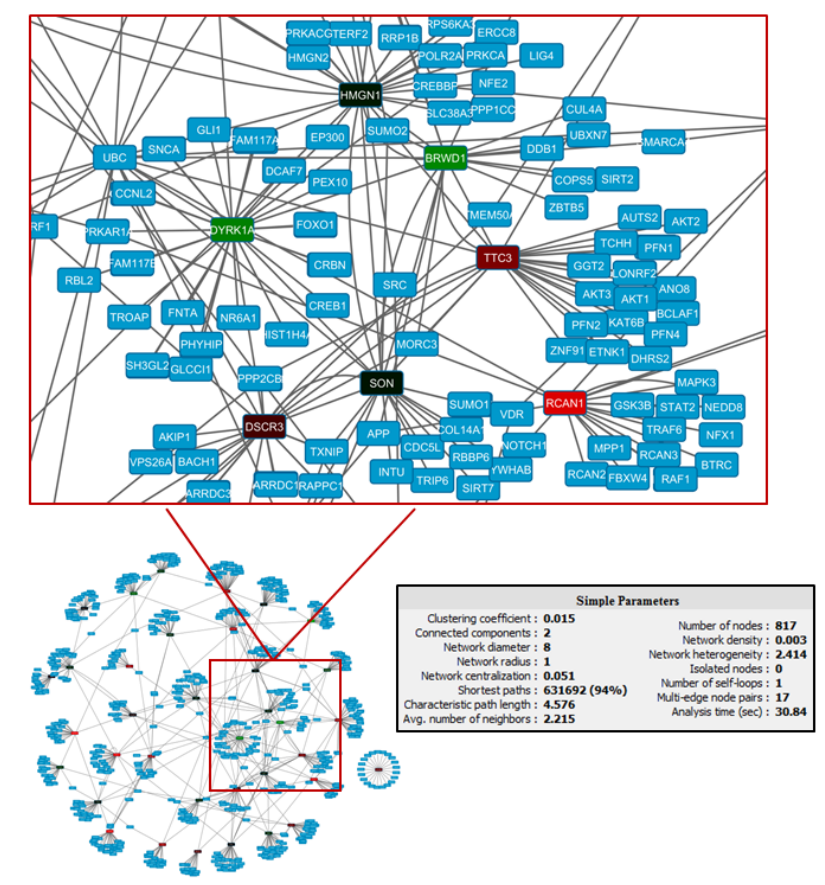

Figure 4. Network interaction of 36 proteins with other DSCR proteins contained in different databases.

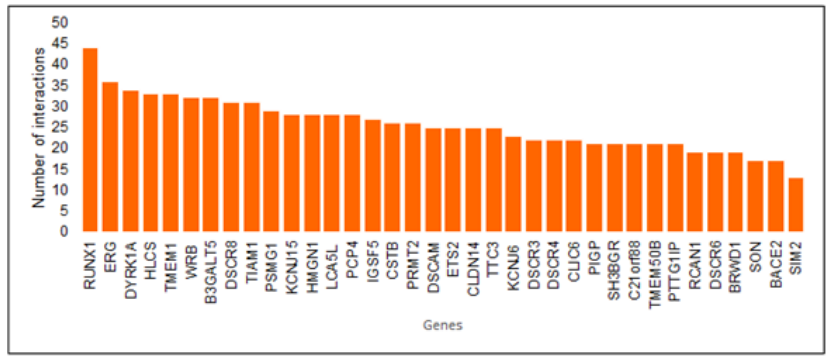

Figure 5. Number of interactions established by each of the 36 DSCR proteins within the network.

Annex 1. Names and symbols of different substructures in limbic lobe and cerebral nuclei. The names were extracted from the Allen Institute for Brain Sciences.

\begin{tabular}{ll}
\hline Structure name & Symbol \\
\hline Brain nuclei & \\
\hline Caudate nuclei head-left & CNH-Left \\
\hline Caudate nuclei head-right & CNH-Right \\
\hline Caudate nuclei tail-left & CNT-Left \\
\hline Caudate nuclei tail-right & CNT-Right \\
\hline Putamen left & Pu-Left \\
\hline Putamen right & Pu-Right
\end{tabular}

\begin{tabular}{|c|c|}
\hline Nucleus accumulus left & NA-Left \\
\hline Nucleus accumulus right & NA-Right \\
\hline Globus pallidum external left & GPE-Left \\
\hline Globus pallidum external right & GPE-Right \\
\hline Globus pallidum internal left & GPI-Left \\
\hline Globus pallidum internal right & GPI-Right \\
\hline Septal nuclei left & SptN-Left \\
\hline Septal nuclei right & SptN-Right \\
\hline Basal forebrain substantia innominata left & BFbSI-Left \\
\hline Basal forebrain substantia innominata right & BFbSI-Right \\
\hline Claustrum left & C-Left \\
\hline Claustrum right & C-Right \\
\hline Amigdala transition zone & ATZ \\
\hline Amigdala basolateral left & ABL-Left \\
\hline Amigdala basomedia right & ABM-Right \\
\hline Central amigdala nucleus & ACN \\
\hline Corto medial amigdala & $\mathrm{ACM}$ \\
\hline Left nucleus amigdala & ALN \\
\hline \multicolumn{2}{|l|}{ Limbic lobe } \\
\hline Short insular gyrus & SIG \\
\hline Cyngulate girus, frontal part & $\mathrm{CgGf}$ \\
\hline Cyngulate girus, parietal part & CgG-Parietal \\
\hline Dental gyrus left & DG-Left \\
\hline Central area 1 left & CA1-Left \\
\hline Central area 1 right & CA-1 Right \\
\hline Central area 2 left & CA2-Left \\
\hline Central area 2 right & CA-2 Right \\
\hline Central area 3 left & CA3-Left \\
\hline Central area 3 right & CA-3 Right \\
\hline Central area 4 left & CA-4-Left \\
\hline Central area 4 right & CA-4 Right \\
\hline Subiculum left & S-Left \\
\hline Subiculum right & S-Right \\
\hline Parahipocampus gyrus left & PHG-Left \\
\hline Parahipocampus gyrus -cos-left & PHG-cos-Left \\
\hline Parahipocampus gyrus right & PHG-Right \\
\hline Parahipocampus gyrus -cos-right & PHG-cos-Righ \\
\hline
\end{tabular}


Fajardo/Vinasco/Montoya/Satizabal/Sanchez/García-Vallejo

Annex 2. Standard values (z score) of the expression levels through 24 substructures of the brain nuclei and 18 from the limbic lobe.

\begin{tabular}{|c|c|c|c|c|c|c|c|c|c|c|}
\hline Gene & Common & Entrez ID & SIG & CgGf & CgG-Par & DG-Left & CA1-Left & CA-1-Right & CA2-left & CA-2 Right \\
\hline RCAN1 & NM_004414.5 & 1827 & 0.139 & -0.3595 & -0.228 & -1.03 & 0.149 & 0.491 & 0.213 & 0.703 \\
\hline PSMG1 & NM_203433.1 & 8624 & 1.131 & 0.2285 & -2.309 & 1.292 & 0.513 & 0.478 & 0.692 & 0.96 \\
\hline DSCR3 & NM_006052.1 & 10311 & 1.018 & -0.2485 & -0.233 & 0.711 & 1.29 & 0.705 & 2.176 & 0.883 \\
\hline DSCR4 & NM_005867.2 & 10281 & 1.436 & -0.2535 & 1.096 & -0.25 & 0.892 & 0.411 & 0.557 & -0.383 \\
\hline DSCR6 & NM_018962.1 & 53820 & 0.572 & -0.249 & 0.43 & 0.764 & 0.993 & 0.174 & 0.781 & 0.928 \\
\hline DSCR8 & NR_026838.1 & 84677 & 0.387 & -0.291 & 1.114 & 0.844 & 1.037 & 1.204 & 0.371 & 1.292 \\
\hline PIGP & NM_153681.1 & 51227 & 0.373 & -0.149 & -2.107 & 2.122 & 0.217 & 0.122 & 0.662 & 0.467 \\
\hline CLIC6 & NM_053277.1 & 54102 & 0.09 & -0.316 & 0.563 & -1.035 & 2.807 & 2.411 & 0.433 & 0.891 \\
\hline$B A C E 2$ & NM_012105.3 & 25825 & -2.501 & 0.0305 & -0.676 & -2.431 & 0.359 & 0.614 & -0.189 & -1.031 \\
\hline$B R W D 1$ & NM_018963.3 & 54014 & 0.08 & 0.0605 & 0.095 & 1.178 & 0.719 & -0.654 & -0.007 & 0.307 \\
\hline DSCAM & NM_001389.3 & 1826 & 0.836 & 0.011 & 0.189 & 0.79 & 0.913 & -0.216 & 0.427 & 0.023 \\
\hline DYRK1A & NM_130438.1 & 1859 & -0.101 & 0.5235 & 0.503 & 0.663 & 1.946 & 0.385 & 0.32 & 1.257 \\
\hline$E R G$ & NM_001136154.1 & 2078 & -0.204 & -0.341 & 0.072 & -0.643 & 0.56 & 0.485 & -0.772 & -1.711 \\
\hline ETS2 & NM_005239.4 & 2114 & -0.427 & -0.501 & 0.145 & 0.889 & 0.381 & -0.193 & -0.168 & -0.625 \\
\hline KCNJ6 & NM_002240.2 & 3763 & 1.76 & 0.257 & 0.086 & 1.574 & 1.393 & 1.096 & 1.257 & 1.932 \\
\hline$R U N \times 1$ & NM_001122607.1 & 861 & 0.387 & -0.571 & -0.101 & -1.355 & 0.679 & -0.473 & -1.171 & -0.701 \\
\hline$S H 3 B G R$ & $\begin{array}{l}\text { NM_001001713. } \\
1\end{array}$ & 6450 & 1.151 & 0.7375 & -0.112 & 0.185 & 0.747 & 0.367 & 0.48 & 1.069 \\
\hline SIM2 & NM_005069.2 & 6493 & 0.306 & -0.365 & -0.105 & -1.081 & 0.279 & -0.674 & -0.37 & -1.133 \\
\hline CLDN14 & NM_012130.2 & 23562 & -0.012 & 0.107 & -0.283 & 0.126 & 0.486 & 0.911 & -0.223 & 0.458 \\
\hline TTC3 & $\begin{array}{l}\text { NM_} \\
1\end{array}$ & 7267 & 0.437 & 0.551 & 0.465 & 1.175 & 0.930 & 0.751 & 1.810 & 1.811 \\
\hline SON & NM_138927.1 & 6651 & 0.162 & -0.184 & -0.023 & 0.489 & 0.020 & 0.072 & -0.182 & -0.535 \\
\hline HLCS & NM_000411.4 & 3141 & 0.179 & -0.257 & 0.300 & 1.808 & 1.033 & 0.599 & 0.771 & 0.186 \\
\hline KCNJ15 & NM_002243.3 & 3772 & -0.668 & 0.453 & -0.081 & 0.010 & 0.487 & 1.387 & -0.419 & 0.777 \\
\hline HMGN1 & NM_004965.6 & 3150 & -0.345 & -0.094 & -0.328 & 0.530 & -0.139 & -0.494 & -0.276 & -1.138 \\
\hline$W R B$ & NM_004627.4 & 7485 & 0.401 & 0.699 & -0.782 & -0.269 & -0.814 & -1.034 & -0.582 & -0.443 \\
\hline$\angle C A 5 L$ & NM_152505.2 & 150082 & 0.224 & 0.088 & 1.671 & -0.057 & -0.512 & 1.029 & -0.221 & 0.520 \\
\hline C21orf88 & NR_026542.1 & 114041 & -0.480 & -0.039 & -0.959 & 0.507 & 1.034 & 1.153 & 0.938 & 1.449 \\
\hline B3GALT5 & NM_033173.1 & 10317 & -0.776 & 0.138 & -0.924 & 0.257 & 0.900 & 0.980 & -0.099 & 1.172 \\
\hline TMEM1 & NM_003274.3 & 7109 & -0.627 & -0.313 & -0.645 & 0.433 & 0.681 & 0.001 & -0.051 & -0.205 \\
\hline IGSF5 & $\begin{array}{l}\text { NM_001080444. } \\
1\end{array}$ & 150084 & -0.828 & 0.078 & -1.009 & -0.323 & -0.010 & 0.871 & 0.202 & 0.930 \\
\hline PCP4 & NM_006198.2 & 5121 & -0.237 & -0.532 & -0.822 & -0.042 & 0.263 & -1.461 & -0.538 & -1.004 \\
\hline CSTB & NM_000100.2 & 1476 & 0.202 & 0.618 & -0.271 & -1.553 & -0.379 & -0.754 & -1.051 & -1.122 \\
\hline TMEM50B & NM_006134.4 & 757 & -0.144 & -0.230 & -0.464 & -0.858 & -0.984 & -1.133 & -0.718 & -0.107 \\
\hline PTTG1IP & NM_004339.2 & 754 & 0.741 & 0.070 & -0.814 & -0.563 & -0.999 & -1.028 & -0.975 & -1.228 \\
\hline TIAM1 & NM_003253.1 & 7074 & -0.849 & -0.899 & -0.171 & 2.290 & -0.643 & -0.706 & 0.566 & -0.099 \\
\hline PRMT2 & NM_206962.1 & 3275 & -0.013 & -0.110 & -0.430 & -0.171 & -0.751 & -0.905 & -0.544 & -0.639 \\
\hline
\end{tabular}


Complex networks of interaction of genes located in the critical region of Down syndrome expressed in the normal human brain

\begin{tabular}{|c|c|c|c|c|c|c|c|c|c|c|}
\hline Gene & Common & Entrez ID & CA3-left & CA-3 Right & CA4-left & CA-4 Right & S-Left & S-Right & PHG-L & PHG-cos-L \\
\hline RCAN1 & NM_004414.5 & 1827 & 0.056 & 0.106 & 2.641 & -0.137 & 0.993 & 0.888 & 1.169 & 0.129 \\
\hline PSMG1 & NM_203433.1 & 8624 & 1.166 & 1.28 & 2.823 & -1.401 & 0.636 & 0.083 & 1.117 & 0.361 \\
\hline DSCR3 & NM_006052.1 & 10311 & 0.204 & 0.484 & 0.808 & -0.976 & 0.001 & 0.561 & 0.909 & -0.65 \\
\hline DSCR4 & NM_005867.2 & 10281 & 0.373 & -0.008 & 2.99 & -0.104 & -1.547 & -0.703 & 2.645 & 1.119 \\
\hline DSCR6 & NM_018962.1 & 53820 & 0.788 & 0.151 & 2.237 & 1.182 & 0.363 & -1.39 & 0.537 & 0.037 \\
\hline DSCR8 & NR_026838.1 & 84677 & 1.743 & 0.886 & 2.721 & 0.713 & 0.323 & 1.434 & 0.281 & 1.503 \\
\hline PIGP & NM_153681.1 & 51227 & 1.587 & 0.349 & 1.877 & 2.006 & 0.483 & 0.479 & 2.774 & 0.311 \\
\hline CLIC6 & NM_053277.1 & 54102 & -0.285 & -0.627 & 0.37 & -0.175 & 1.233 & 1.134 & 1.951 & 1.908 \\
\hline BACE2 & NM_012105.3 & 25825 & -1.167 & -1.363 & 0.975 & -0.495 & -0.757 & -0.471 & -0.823 & -0.755 \\
\hline BRWD1 & NM_018963.3 & 54014 & 0.357 & 0.213 & -1.598 & -0.154 & 0.048 & -0.003 & 1.129 & -0.24 \\
\hline DSCAM & NM_001389.3 & 1826 & 0.494 & -0.249 & 1.917 & 1.463 & 0.421 & 0.486 & 1.517 & 1.064 \\
\hline DYRK1A & NM_130438.1 & 1859 & -0.093 & 0.687 & -1.506 & -1.329 & 0.504 & 0.053 & -0.078 & 0.348 \\
\hline$E R G$ & NM_001136154.1 & 2078 & 0.197 & -0.49 & 1.694 & 0.293 & 0.027 & -0.761 & 0.983 & 0.493 \\
\hline ETS2 & NM_005239.4 & 2114 & -0.689 & -0.923 & -0.125 & 0.247 & 0.754 & 0.531 & -0.075 & -0.078 \\
\hline KCNJ6 & NM_002240.2 & 3763 & 1.05 & 1.2 & -1.101 & 1.01 & 0.159 & 0.398 & 0.377 & 0.3 \\
\hline$R U N X 1$ & NM_001122607.1 & 861 & -0.713 & -0.173 & 1.753 & 0.657 & -0.173 & -0.57 & 0.705 & -0.035 \\
\hline$S H 3 B G R$ & $\underset{1}{\text { NM_}}$ 001001713. & 6450 & 0.35 & 0.561 & 0.273 & 0.44 & 0.144 & -0.024 & 0.954 & 0.926 \\
\hline SIM2 & NM_005069.2 & 6493 & -0.09 & 0.011 & 1.959 & 0.76 & 0.565 & -0.792 & 0.715 & 0.193 \\
\hline CLDN14 & NM_012130.2 & 23562 & 0.983 & -0.230 & -0.532 & 0.265 & 0.403 & -0.355 & 0.470 & 1.218 \\
\hline TTC3 & $\underset{1}{\text { NM_001001894. }}$ & 7267 & 1.744 & 1.642 & 1.470 & 1.470 & 0.336 & 0.494 & 0.924 & 0.849 \\
\hline SON & NM_138927.1 & 6651 & -0.581 & -0.381 & -0.230 & -0.475 & -0.258 & -0.183 & -0.138 & -0.564 \\
\hline HLCS & NM_000411.4 & 3141 & -0.086 & -0.457 & -0.123 & -0.451 & 0.138 & -0.505 & 0.297 & -0.774 \\
\hline KCNJ15 & NM_002243.3 & 3772 & 1.133 & -0.131 & -0.558 & 0.282 & 0.304 & -0.489 & 0.182 & 0.735 \\
\hline HMGN1 & NM_004965.6 & 3150 & -0.556 & -0.821 & -0.288 & -0.455 & -0.303 & -0.400 & 0.266 & 0.471 \\
\hline$W R B$ & NM_004627.4 & 7485 & -1.105 & 0.111 & -0.242 & -0.748 & -1.128 & -0.129 & 0.578 & 1.933 \\
\hline$\angle C A 5 L$ & NM_152505.2 & 150082 & 0.270 & -0.424 & -0.362 & -0.354 & -0.480 & -0.504 & 0.265 & 1.114 \\
\hline C21orf88 & NR_026542.1 & 114041 & 1.453 & 1.421 & 1.167 & 1.509 & -0.278 & -0.323 & -0.132 & 0.387 \\
\hline B3GALT5 & NM_033173.1 & 10317 & 1.402 & 0.127 & 0.081 & 0.265 & 0.181 & -0.354 & 0.271 & 0.147 \\
\hline TMEM1 & NM_003274.3 & 7109 & 0.709 & -0.091 & 0.030 & 0.258 & -0.243 & -0.436 & 0.456 & -0.972 \\
\hline IGSF5 & $\underset{1}{\text { NM_001080444. }}$ & 150084 & 0.089 & 0.623 & 1.201 & -0.136 & -0.484 & -0.805 & -0.884 & -0.324 \\
\hline PCP4 & NM_006198.2 & 5121 & -2.043 & -2.133 & -1.909 & -1.466 & 0.928 & 0.411 & -1.056 & -1.272 \\
\hline CSTB & NM_000100.2 & 1476 & -0.686 & -1.037 & -0.192 & -1.277 & -0.084 & -0.146 & 0.895 & 1.200 \\
\hline TMEM50B & NM_006134.4 & 757 & -1.411 & -0.288 & -0.602 & -0.898 & -0.415 & -0.669 & -0.295 & -0.223 \\
\hline PTTG1IP & NM_004339.2 & 754 & -0.924 & -0.868 & -0.354 & -0.701 & -0.977 & -0.747 & 1.681 & 0.642 \\
\hline TIAM1 & NM_003253.1 & 7074 & -0.279 & -0.631 & -0.479 & -0.096 & 0.020 & -0.422 & -0.525 & -0.215 \\
\hline PRMT2 & NM_206962.1 & 3275 & -0.825 & -0.706 & -0.407 & -0.743 & -0.364 & -0.496 & 0.439 & 0.351 \\
\hline
\end{tabular}




\begin{tabular}{|c|c|c|c|c|c|c|c|c|c|c|}
\hline Gene & Common & Entrez ID & PHG-R & PHG-cos-R & CNH-Left & CNH-Right & CNT-Left & CNT-Right & Pu-Left & Pu-Right \\
\hline RCAN1 & NM_004414.5 & 1827 & 0.261 & 0.931 & 1.112 & 1.433 & 0.699 & 1.525 & 2.827 & 2.66 \\
\hline PSMG1 & NM_203433.1 & 8624 & -0.305 & 0.457 & -0.589 & -1.794 & -0.8 & -2.495 & -1.758 & -2.499 \\
\hline DSCR3 & NM_006052.1 & 10311 & -0.363 & -0.393 & 0.707 & -0.423 & -0.716 & -0.079 & -0.19 & -0.601 \\
\hline DSCR4 & NM_005867.2 & 10281 & -0.354 & -0.031 & -0.433 & 0.216 & 0.322 & 0.886 & -0.271 & 1.416 \\
\hline DSCR6 & NM_018962.1 & 53820 & -0.238 & -0.233 & 1.281 & 2.312 & 2.049 & 2.619 & 3 & 2.3 \\
\hline DSCR8 & NR_026838.1 & 84677 & 0.095 & 1.576 & -0.749 & 0.793 & 0.268 & 0.522 & 0.874 & 1.463 \\
\hline PIGP & NM_153681.1 & 51227 & -1.419 & 0.108 & 0.306 & -0.548 & 0.821 & 1.478 & 0.632 & -1.044 \\
\hline CLIC6 & NM_053277.1 & 54102 & 2.147 & 1.22 & 0.849 & -0.397 & 1.12 & 0.861 & 0.382 & 0.375 \\
\hline BACE2 & NM_012105.3 & 25825 & -1 & -0.327 & 0.169 & -0.164 & -0.604 & -0.524 & 0.077 & 0.436 \\
\hline$B R W D 1$ & NM_018963.3 & 54014 & 0.244 & 0.179 & -0.403 & -0.196 & 0.259 & 0.013 & -0.132 & -0.059 \\
\hline DSCAM & NM_001389.3 & 1826 & 0.475 & 0.693 & -0.643 & -0.1475 & -1.899 & -1.938 & 0.48 & 1.086 \\
\hline DYRK1A & NM_130438.1 & 1859 & 0.304 & 0.268 & -0.94 & -1.861 & 0.056 & 0.018 & -1.485 & -1.604 \\
\hline$E R G$ & NM_001136154.1 & 2078 & 0.594 & -0.424 & -0.025 & 0.317 & -0.183 & 0.088 & 1.575 & 0.86 \\
\hline ETS2 & NM_005239.4 & 2114 & -0.417 & -0.085 & -0.952 & -1.439 & -1.118 & -0.995 & -1.09 & -0.997 \\
\hline KCNJ6 & NM_002240.2 & 3763 & 0.489 & 0.165 & -2.365 & -2.283 & -1.931 & -2.37 & -2.403 & -2.162 \\
\hline$R U N X 1$ & NM_001122607.1 & 861 & -0.179 & -0.555 & 1.408 & 1.79 & 0.886 & 1.165 & 1.257 & 1.402 \\
\hline$S H 3 B G R$ & $\underset{1}{\text { NM_001001713. }}$ & 6450 & 0.59 & 0.364 & 0.75 & 0.965 & -0.142 & 0.26 & 1.005 & 1.154 \\
\hline SIM2 & NM_005069.2 & 6493 & -0.164 & -0.608 & 0.02 & 0.645 & 0.055 & -0.27 & 0.736 & 0.678 \\
\hline CLDN14 & NM_012130.2 & 23562 & 0.797 & 0.035 & 0.285 & 0.633 & 0.595 & 0.236 & 0.196 & 0.637 \\
\hline TTC3 & $\begin{array}{l}\text { NM_001001894. } \\
1\end{array}$ & 7267 & 1.949 & 0.823 & -0.938 & -0.508 & 0.973 & -0.061 & -0.265 & -0.403 \\
\hline SON & NM_138927.1 & 6651 & 0.339 & -0.493 & 0.478 & 0.242 & 0.011 & 0.041 & 0.932 & 0.608 \\
\hline HLCS & NM_000411.4 & 3141 & 0.793 & -0.003 & 2.027 & 2.049 & 0.505 & -0.759 & 1.854 & 2.089 \\
\hline KCNJ15 & NM_002243.3 & 3772 & 0.849 & -0.073 & 0.268 & 0.553 & 0.631 & -0.143 & 0.099 & 0.442 \\
\hline HMGN1 & NM_004965.6 & 3150 & 0.781 & -0.015 & 0.664 & 1.308 & 0.438 & 0.336 & 0.605 & 0.804 \\
\hline$W R B$ & NM_004627.4 & 7485 & 0.776 & -0.265 & -0.032 & -0.433 & -1.818 & -0.729 & 0.424 & 0.563 \\
\hline LCA5L & NM_152505.2 & 150082 & 0.473 & 0.518 & 1.770 & 0.294 & 1.005 & 0.164 & 0.579 & 1.030 \\
\hline C21orf88 & NR_026542.1 & 114041 & 0.040 & 0.782 & 2.238 & 3.418 & 1.441 & 0.966 & 2.863 & 2.942 \\
\hline B3GALT5 & NM_033173.1 & 10317 & 0.180 & 0.505 & 2.611 & 3.416 & 0.951 & 0.983 & 2.646 & 3.120 \\
\hline TMEM1 & NM_003274.3 & 7109 & -0.206 & -0.284 & 0.654 & 0.979 & 0.090 & -0.121 & 1.299 & 0.887 \\
\hline IGSF5 & $\begin{array}{l}\text { NM_001080444. } \\
1\end{array}$ & 150084 & -1.103 & -0.869 & 2.376 & 2.394 & 0.897 & 1.149 & 2.617 & 2.648 \\
\hline PCP4 & NM_006198.2 & 5121 & -1.141 & -0.147 & 3.066 & 3.867 & 0.677 & 1.410 & 3.414 & 3.575 \\
\hline CSTB & NM_000100.2 & 1476 & -0.054 & 0.944 & 1.688 & 1.104 & -0.759 & 0.179 & 0.859 & 0.858 \\
\hline TMEM50B & NM_006134.4 & 757 & -0.192 & -0.570 & 0.167 & 0.230 & -1.636 & -1.064 & 0.570 & 0.496 \\
\hline PTTG1IP & NM_004339.2 & 754 & 0.617 & 0.684 & 1.041 & 1.404 & -0.454 & -0.357 & 1.411 & 1.608 \\
\hline TIAM1 & NM_003253.1 & 7074 & -0.251 & -0.431 & 0.295 & 0.396 & 0.076 & 0.556 & -0.180 & -0.009 \\
\hline PRMT2 & NM_206962.1 & 3275 & 0.030 & 0.157 & -1.436 & -1.944 & -1.538 & -1.240 & -1.926 & -2.274 \\
\hline
\end{tabular}


Complex networks of interaction of genes located in the critical region of Down syndrome expressed in the normal human brain

\begin{tabular}{|c|c|c|c|c|c|c|c|c|c|c|}
\hline Gene & Common & Entrez ID & NA-Left & NA-Right & GPE-Left & GPE-Right & GPI-Left & GPI-Right & SptN-Left & SptN-Right \\
\hline RCAN1 & NM_004414.5 & 1827 & 0.518 & 0.353 & 2.669 & 2.12 & 1.656 & 1.154 & 0.158 & 0.062 \\
\hline PSMG1 & NM_203433.1 & 8624 & -0.198 & -0.237 & -1.912 & -1.563 & -1.507 & -0.453 & -0.304 & -0.553 \\
\hline DSCR3 & NM_006052.1 & 10311 & -1.337 & -0.935 & 1.089 & 0.614 & -0.215 & 0.1 & -0.095 & -1.509 \\
\hline DSCR4 & NM_005867.2 & 10281 & 1.824 & 0.04 & -0.064 & -0.09 & 0.656 & 0.66 & 0.29 & 0.061 \\
\hline DSCR6 & NM_018962.1 & 53820 & -1.268 & -1.834 & 0.102 & 0.174 & 0.467 & -1.081 & -0.134 & -0.063 \\
\hline DSCR8 & NR_026838.1 & 84677 & 0.724 & -0.348 & 0.543 & 0.748 & 0.524 & 1.272 & 0.507 & 0.519 \\
\hline PIGP & NM_153681.1 & 51227 & 1.971 & 0.633 & 1.055 & 1.131 & 1.101 & 1.357 & 0.029 & -0.255 \\
\hline CLIC6 & NM_053277.1 & 54102 & -0.692 & 0.335 & 1.797 & 0.752 & 0.166 & 0.208 & -0.66 & -0.354 \\
\hline BACE2 & NM_012105.3 & 25825 & -0.457 & 0.258 & 2.444 & 2.176 & 2.367 & 1.661 & 0.669 & 0.796 \\
\hline$B R W D 1$ & NM_018963.3 & 54014 & 0.282 & 0.369 & -0.644 & -0.429 & -0.887 & -0.938 & 0.285 & 0.187 \\
\hline DSCAM & NM_001389.3 & 1826 & 0.493 & 0.662 & 0.107 & 0.637 & 1.049 & 0.804 & 0.25 & 0.731 \\
\hline DYRK1A & NM_130438.1 & 1859 & -0.328 & -0.616 & -1.358 & -1.694 & -0.495 & -1.305 & -0.309 & -0.754 \\
\hline$E R G$ & NM_001136154.1 & 2078 & -0.372 & 0.465 & 2.089 & 1.617 & 2.218 & 1.03 & -0.352 & 0.375 \\
\hline ETS2 & NM_005239.4 & 2114 & 0.665 & 0.517 & -1.152 & -1.27 & -0.705 & -0.126 & -0.38 & -0.544 \\
\hline KCNJ6 & NM_002240.2 & 3763 & -0.618 & -0.177 & -1.778 & -1.569 & -1.531 & -0.836 & 0.196 & -0.095 \\
\hline RUNX1 & NM_001122607.1 & 861 & 0.286 & 0.49 & 0.687 & 1.482 & 1.719 & 1.638 & 0.257 & 1.002 \\
\hline$S H 3 B G R$ & $\underset{1}{\text { NM_}}$ 001001713. & 6450 & 0.833 & 0.688 & 0.77 & 1.241 & 0.718 & 0.631 & -0.445 & 0.195 \\
\hline SIM2 & NM_005069.2 & 6493 & 0.285 & -0.461 & 0.983 & 1.313 & 1.155 & 0.416 & 0.352 & -0.166 \\
\hline CLDN14 & NM_012130.2 & 23562 & 0.019 & -0.507 & 0.663 & 0.971 & 0.406 & 0.582 & 0.294 & -0.088 \\
\hline TTC3 & $\underset{1}{\text { NM_}}{ }_{1} 001001894$. & 7267 & 0.028 & -0.539 & -1.828 & -1.589 & -1.753 & -1.217 & -0.580 & -0.336 \\
\hline SON & NM_138927.1 & 6651 & 0.119 & 0.864 & 0.879 & 1.192 & 0.904 & 1.349 & 0.687 & 0.047 \\
\hline HLCS & NM_000411.4 & 3141 & 1.349 & 0.169 & 1.705 & 1.717 & 2.981 & 1.134 & 0.286 & -0.004 \\
\hline KCNJ15 & NM_002243.3 & 3772 & 0.119 & 0.118 & 0.683 & 0.197 & 0.630 & -0.047 & -0.126 & 0.794 \\
\hline HMGN1 & NM_004965.6 & 3150 & 0.849 & 0.944 & 0.793 & 0.885 & 1.170 & 0.843 & 0.766 & 0.765 \\
\hline$W R B$ & NM_004627.4 & 7485 & 0.297 & 0.532 & 0.955 & 1.493 & 1.088 & 0.486 & 0.224 & -0.309 \\
\hline LCA5L & NM_152505.2 & 150082 & 0.359 & 0.152 & 0.673 & 0.551 & 0.767 & -0.553 & 0.565 & 0.346 \\
\hline C21orf88 & NR_026542.1 & 114041 & 1.415 & 1.150 & 0.893 & 0.536 & 1.441 & -0.358 & 0.029 & 0.674 \\
\hline B3GALT5 & NM_033173.1 & 10317 & 0.574 & 0.258 & -0.012 & -0.198 & 0.446 & -0.474 & 0.333 & 1.056 \\
\hline TMEM1 & NM_003274.3 & 7109 & -0.146 & -0.854 & 1.312 & 1.226 & 1.922 & 0.611 & 0.184 & 0.190 \\
\hline IGSF5 & $\underset{1}{\text { NM_001080444. }}$ & 150084 & 1.273 & 0.944 & 1.142 & 1.000 & 1.552 & -0.189 & 0.814 & -0.224 \\
\hline PCP4 & NM_006198.2 & 5121 & 1.689 & 1.727 & 0.917 & 0.822 & 0.121 & 0.419 & 0.268 & 0.509 \\
\hline CSTB & NM_000100.2 & 1476 & 0.445 & 0.752 & 1.407 & 1.522 & 2.112 & 1.083 & 0.509 & -0.118 \\
\hline TMEM50B & NM_006134.4 & 757 & 0.235 & 0.661 & 0.932 & 1.167 & 1.186 & -0.244 & 0.595 & -0.293 \\
\hline PTTG1IP & NM_004339.2 & 754 & 0.547 & 0.144 & 2.952 & 2.643 & 3.572 & 2.054 & 1.049 & 0.600 \\
\hline TIAM1 & NM_003253.1 & 7074 & 0.576 & 0.324 & 0.104 & -0.381 & -0.655 & -0.637 & -0.011 & 0.154 \\
\hline PRMT2 & NM_206962.1 & 3275 & -0.156 & 0.382 & 0.693 & 0.768 & 0.438 & 0.725 & 0.510 & 0.228 \\
\hline
\end{tabular}




\begin{tabular}{|c|c|c|c|c|c|c|c|c|c|c|c|c|}
\hline Gene & Common & Entrez ID & BFbSI-L & BFbSI-R & C-Left & C-Right & ATZ & ABL-Left & ABM-Left & $\mathrm{ACN}$ & $\mathrm{ACM}$ & ALN \\
\hline RCAN1 & NM_004414.5 & 1827 & 0.339 & 0.888 & -1.268 & -0.295 & -0.696 & -0.202 & 0.105 & -0.432 & 0.163 & -0.031 \\
\hline PSMG1 & NM_203433.1 & 8624 & -0.357 & -1.262 & -0.008 & -0.075 & 0.427 & 0.715 & -1.349 & 0.082 & -0.4 & -0.157 \\
\hline DSCR3 & NM_006052.1 & 10311 & 0.069 & 0.247 & -1.554 & -0.976 & -0.597 & -1.358 & 0.187 & 0.901 & -1.281 & -0.398 \\
\hline DSCR4 & NM_005867.2 & 10281 & -0.48 & -0.274 & 0.785 & 0.542 & -0.79 & -0.704 & 0.126 & -0.453 & 0.198 & -1.022 \\
\hline DSCR6 & NM_018962.1 & 53820 & 0.318 & -0.471 & 1.397 & 0.668 & -0.565 & -0.538 & -0.18 & -0.429 & 0.508 & -0.987 \\
\hline DSCR8 & NR_026838.1 & 84677 & -0.366 & -0.358 & 1.896 & 0.677 & 0.413 & 0.528 & 0.434 & -0.211 & 0.662 & -1.128 \\
\hline PIGP & NM_153681.1 & 51227 & -0.397 & -0.122 & -3 & -0.34 & 0.497 & -0.453 & -0.921 & -0.084 & -0.936 & -0.343 \\
\hline CLIC6 & NM_053277.1 & 54102 & 0.001 & -0.472 & 0.549 & -0.4 & 0.787 & -0.402 & -0.015 & -0.721 & -0.124 & 0.032 \\
\hline BACE2 & NM_012105.3 & 25825 & 0.962 & -0.024 & -0.803 & 0.42 & -1.092 & -1.61 & -0.81 & -0.688 & -0.957 & 0.158 \\
\hline$B R W D 1$ & NM_018963.3 & 54014 & -0.492 & -0.269 & 0.381 & 0.861 & 0.858 & 0.918 & 0.678 & 0.343 & 0.288 & 0.117 \\
\hline DSCAM & NM_001389.3 & 1826 & 0.908 & 0.67 & 1.005 & 0.999 & -0.687 & -0.165 & 1.202 & -0.438 & 0.201 & -0.029 \\
\hline DYRK1A & NM_130438.1 & 1859 & -0.648 & -0.661 & -0.782 & 0.348 & 0.788 & 0.578 & -0.481 & 0.939 & -0.231 & -0.02 \\
\hline$E R G$ & NM_001136154.1 & 2078 & -1.04 & 0.744 & 0.812 & 0.873 & -0.759 & -0.969 & 0.073 & -1.195 & 0.461 & -0.659 \\
\hline ETS2 & NM_005239.4 & 2114 & -1.182 & -0.191 & 0.859 & 0.392 & -0.01 & 0.082 & 0.462 & -0.672 & 0.901 & 0.623 \\
\hline KCNJ6 & NM_002240.2 & 3763 & -1.357 & -1.297 & -0.526 & -0.427 & 0.089 & 0.387 & -0.279 & 1.319 & -0.097 & -0.565 \\
\hline RUNX1 & NM_001122607.1 & 861 & -0.387 & -0.245 & 1.299 & 0.532 & 0.389 & -0.623 & 0.126 & -1.933 & -0.105 & 0.384 \\
\hline$S H 3 B G R$ & ${ }_{1}^{\text {NM_001001713. }}$ & 6450 & 0.014 & 0.715 & 0.224 & 0.013 & 0.739 & 0.855 & 1.876 & -0.094 & -0.38 & 0.065 \\
\hline SIM2 & NM_005069.2 & 6493 & -0.112 & 0.797 & 0.863 & 1.395 & -0.327 & -0.424 & 0.278 & -1.618 & 0.449 & 0.704 \\
\hline CLDN14 & NM_012130.2 & 23562 & -0.457 & -0.435 & 2.331 & 1.152 & 0.665 & 1.782 & 2.665 & 0.421 & 0.119 & -0.366 \\
\hline TTC3 & ${ }_{1}^{\text {NM_}}$ _001001894. & 7267 & -0.387 & -0.479 & 0.033 & -0.076 & -0.102 & 0.633 & 0.038 & -0.419 & -0.341 & 0.501 \\
\hline SON & NM_138927.1 & 6651 & -0.131 & -0.471 & -0.180 & -0.024 & 0.161 & -0.226 & -0.038 & -0.060 & -0.424 & -0.455 \\
\hline HLCS & NM_000411.4 & 3141 & -0.659 & 0.685 & -1.000 & -0.859 & -0.164 & -0.043 & -0.942 & -1.009 & -1.019 & -2.202 \\
\hline KCNJ15 & NM_002243.3 & 3772 & -0.831 & 0.222 & -0.155 & 0.149 & 0.270 & -0.152 & -0.569 & 0.159 & -0.059 & -0.527 \\
\hline HMGN1 & NM_004965.6 & 3150 & 0.619 & -0.296 & 0.035 & -0.482 & 0.977 & 0.726 & 0.811 & 0.398 & 0.414 & -0.573 \\
\hline$W R B$ & NM_004627.4 & 7485 & 0.621 & 0.362 & 0.020 & 0.004 & -0.295 & -0.540 & 0.290 & 0.392 & -0.072 & 0.567 \\
\hline LCA5L & NM_152505.2 & 150082 & 0.434 & -0.533 & -0.320 & 0.330 & 0.976 & 0.024 & 0.603 & -0.066 & -0.091 & -0.542 \\
\hline C21orf88 & NR_026542.1 & 114041 & 0.387 & 0.172 & -1.228 & -1.098 & 0.923 & 0.131 & -0.037 & 0.337 & 0.223 & -1.015 \\
\hline B3GALT5 & NM_033173.1 & 10317 & 0.024 & 0.313 & -0.031 & 0.068 & -0.174 & 0.288 & -0.085 & 0.217 & 0.030 & -0.492 \\
\hline TMEM1 & NM_003274.3 & 7109 & -0.372 & -0.344 & 0.889 & 1.019 & 0.262 & -0.133 & -0.219 & -0.180 & -0.322 & 0.048 \\
\hline IGSF5 & $\begin{array}{l}\text { NM_001080444. } \\
1\end{array}$ & 150084 & 0.942 & 0.528 & -1.210 & -1.394 & 0.285 & -0.509 & -0.629 & 0.125 & 0.199 & -1.339 \\
\hline$P C P 4$ & NM_006198.2 & 5121 & 1.241 & 1.255 & -0.416 & -0.324 & 0.104 & -0.071 & -0.639 & 0.253 & -0.788 & -1.824 \\
\hline CSTB & NM_000100.2 & 1476 & 0.953 & 1.087 & 0.600 & 0.044 & 0.630 & 0.286 & 0.127 & 0.816 & 0.131 & -0.481 \\
\hline TMEM50B & NM_006134.4 & 757 & 0.547 & 0.274 & -1.117 & -1.051 & -1.241 & -1.184 & -1.103 & -0.844 & -0.976 & -1.617 \\
\hline PTTG1IP & NM_004339.2 & 754 & 0.253 & 0.097 & 0.048 & -0.169 & 0.390 & 0.109 & 0.425 & 0.094 & 0.172 & -0.106 \\
\hline TIAM1 & NM_003253.1 & 7074 & 0.158 & -0.206 & -1.369 & -1.539 & 0.399 & 0.493 & 0.514 & 0.731 & 0.421 & 0.878 \\
\hline PRMT2 & NM_206962.1 & 3275 & 0.785 & 0.718 & 0.715 & 0.827 & 0.029 & -0.754 & -0.242 & -0.307 & -0.063 & -0.570 \\
\hline
\end{tabular}




\section{Discussion}

Until now, many studies have reported in DS mouse models a generalized overexpression of triplicate genes [26,27]. Thus, other researches on human trisomic tissues showed that only a subset of genes of chromosome 21 are over-expressed related to euploid controls, and the set of genes vary between cell types [28,29], indicating that the presence of three copies of a gene does not necessarily result in a global overexpression.

The expression pattern of a gene provides indirect information about its importance at a functional level, but it also provides information about the area in which it is expressed, correlating the importance of the functional role of this structure for the maintenance of homeostasis and efficiency of brain functioning. Thus, our results provided strong evidence that these areas have a marked gene activity, leading to think that they are sites of vital importance in various biological processes.

The caudate nucleus, putamen and globus pallidus are substructures that had a higher level of expression by the majority of genes in the basal nuclei. The caudate nucleus, takes part in the modulation of motion, just as it has been involved in learning and memory. On the other hand, putamen appears to play an important role in operant conditioning learning through reinforcement. Finally, the globus pallidus receives information from the caudate nucleus and the putamen and sends information to the substantia nigra [30,31]. Its function is to relate maintenance of a basal muscle tone for voluntary movements, carrying out precise activities with hands and feet as well as to keep the body in a specific position $[32,33]$. Moreover, the most expressed areas in the limbic lobe were the central ones and the parahippocampal gyrus, which are closely related substructures in maintaining memory and possible learning centers [34,35]. Studies based on connectivity changes in the brains of patients with DS have shown significant changes in the above-mentioned areas, demonstrating that those are relevant to the emotional and learning processes. Therefore, alterations in the dose of any of these genes in these critical areas would fit with poor cognitive performance of patients with DS [36].

On the other hand, PCP4 also known as protein 4 Purkinje cells, had the highest level of over expression in brain nuclei and the lower sub-expression in the limbic lobe on all genes analysed. The PCP4 (PEP-19) belongs to a family of proteins involved in a signal transduction of calcium modulating through this interaction calmodulin activity. Erhardt et al. suggested that PCP4 protects cells from induced apoptosis, but their exact functions remain unknown yet [12,37]. It is known to be involved in brain development, being present in almost all regions and most abundant in the cerebellum way; besides that it has a very specific expression pattern in adult neurons $[12,38]$. A previous study revealed a high expression of this gene in various brain structures between which the putamen and basal forebrain is included [39], being consistent with the results obtained in this study.
The transgenic mouse models with DS have shown that overexpression of the gene induces PCP4 early neuronal differentiation, in which the additional copy of it, produces a stronger and broader expression in neurons compared with disomic controls. Thus, the highest protein levels have direct consequences in the early stages of differentiation of neurons $[12,40]$. It is important for proper brain function. We can conclude from our results that a large expression related to learning, memory and motor control structures are crucial for both normal adult brain and it becomes a starting point for linking malfunction or overdose of this gene with possible implications for neurological damage in DS or other neuropathology brains.

The KCNJ6 (GIRK2) gene showed great expression in different limbic lobe substructures related to cognitive processes. It is shown as a mediator of different motor functions, playing a key role in learning and memory [41]. DS studies in mice have shown a high over expression in the frontal cortex and hippocampus, and it has been linked in neurogenesis processes and synapses of the latter, suggesting that cognitive disorders, memory and motors in these mice could be related to over expression or malfunction of this gene [42-44].

Additionally, the increased expression of KCNJ6 has been associated with the deterioration in GABAergic function, which appears to contribute to the mechanisms responsible for the cognitive impairments in DS and mice models. This has led to postulate it as a key gene both, in the contribution cognitive deficits and dysfunction of fine motor skills in DS, and possible investigations of gene therapy $[41,44,45]$.

RCAN1 and DSCR2 genes, showed a remarkable expression in many of the studied substructures allowing postulate that these genes are important for the proper functioning of those regions. RCAN1 (DSCR1) presented a remarkable expression in the central areas, the putamen and globus pallidus. Studies have shown that chronic expression of this gene has been associated with several pathologies among which include Alzheimer's disease, cardiac hypertrophy and conditions related to learning and memory in DS associating this with the deficit occurred in the late phase of long-term potentiation [46]. Similarly, the $D S C R 2$ gene showed an opposite expression between brain nuclei and the limbic lobe, with high activity in the latter. It is known that this gene is an important factor associated with DS, expressing in different tissues and fulfilling various functions that are key in regulating the functioning of chromosome 21 [47].

In addition, DYRK1A showed a lot of interactions within the protein network. DYRK1A, is a serine/threonine kinase of dual activity which phosphorylates exogenous substrates on serine/ threonine residues, but it has to autophosphorylate on tyrosine residues $[15,48,49]$. Because of their participation in various structural and functional aspects of the central nervous system, it has been proposed that it could play an important role in the pathogenesis of DS during pregnancy and adult life. These assertions are based on the discovery of a highly characteristic pattern of expression in both fetal and adult tissues [50-52] further that this gene is over expressed in the brains with DS 
and in Ts65Dn mice [50]. Similarly, other experimental data have suggested that this is a sensitive dose gene, for example the variation in gene copies is accompanied by a corresponding variation. It is important to highlight that seven DSCR proteins composing this master node are widely expressed in the brain; they also have in common to be very important for neurogenesis processes. Therefore, it has been observed that the gene RCAN1 interacts with calcineurin $\mathrm{A}$ and inhibits signaling pathways dependent on calcineurin, affecting maybe the central nervous system development. Moreover, it has been found that is over expressed in the brain of fetuses with DS and is involved in the development of Alzheimer's disease in these patients. On the other hand, BRWD1, TTC3 and DSCR3 genes are related to processes of neuronal differentiation, signal transduction and are critical candidates to explain the pathogenesis of DS. Moreover, the HMGN1, BRWD1 and SON genes expressed in brain and interacted with the DYRK1A gene showing a strong correlation between them (group that remains in the heatmap for $H M G N 1$ and $S O N$ genes). It has been observed that these three genes are key for the regulation of cell cycle and apoptotic processes, besides being involved in the assembly of components involved in transcriptional activation $[18,53,54]$.

Based on these results and in addition to the great anatomical correlation with the differential expression of genes DSCR, it is possible to state that the deficit in the dosage of any of these genes in the substructures of the brain nuclei and the limbic lobe (especially in regions with higher expression) can be an extremely important fact in the regulation of their associated functions. This lead to dysfunction of learning, memory and motor skills, which are characteristic, observed in varying degrees in the symptoms of DS [55]. Although it is worth noting that the results obtained come from databases, giving a bioinformatics approach which may propose models of the effect of altering gene dosage [56].

However, the mechanisms involved in the pathogenesis of DS, are still not fully understood, indicating that more studies to elucidate more about the complex biological processes associated with this disorder are needed. Consequently, exploration in databases related to brain gene expression, are a great help to clarify the picture at a genomic and proteomic level, both in normal brain and with DS or other neuropathology.

\section{Declarations}

\section{Ethics approval and consent to participate}

Not applicable

\section{Consent for publication}

Not applicable

\section{Availability of data and materials}

The datasets supporting the conclusions of this article are available in the Human brain atlas repository, (http:// human.brain-map.org/), and its additional file (Dataset).

\section{Disclosure statement}

The authors declare that there is no conflict of interest regarding the publication of this paper.

\section{Funding}

We declared that the Universidad del Valle and the Universidad Autonoma de Occidente financed the design of the study and collection, analysis, and interpretation of data and in writing the manuscript.

\section{Authors' contributions}

DF participated in the acquisition of data, analysis and interpretation of data, and was involved in drafting the manuscript. JRO, FGV and AS participated in the design of the study and performed the statistical analysis. KV, JCM and JMS were involved in drafting the manuscript and its critical review. All authors read and approved the final manuscript.

\section{Acknowledgment}

The current work was financially support by a scientific internal call of the Vice-rectory of Investigations of the Universidad del Valle and the Universidad Autonoma de Occidente, Cali-Colombia.

\section{References}

1. Cannon TD, Keller MC. Endophenotypes in the genetic analyses of mental disorders. Annu Rev Clin Psychol 2006; 2: 267-290.

2. Tulving E. Episodic memory: from mind to brain. Annu Rev Psychol 2002; 53: 1-25.

3. Kelsch W, Sim S, Lois C. Watching synaptogenesis in the adult brain. Annu Rev Neurosci 2010; 33: 131-149.

4. Pascual-Leone A, Amedi A, Fregni F, Merabet LB. The plastic human brain cortex. Annu Rev Neurosci 2005; 28: 377-401.

5. Toga AW, Thompson PM. Genetics of brain structure and intelligence. Annu Rev Neurosci 2005; 28: 1-23.

6. $\mathrm{Hu} \mathrm{WF}$, Chahrour MH, Walsh CA. The diverse genetic landscape of neurodevelopmental disorders. Annu Rev Genomics Hum Genet 2014; 15: 195-213.

7. Yankner BA, Lu T, Loerch P. The aging brain. Annu Rev Pathol 2008; 3: 41-66.

8. Fernald RD. Social control of the brain. Annu Rev Neurosci 2012; 35: 133-151.

9. Ait Yahya-Graison E, Aubert J, Dauphinot L, Rivals I, Prieur M, Golfier G. Classification of human chromosome 21 gene-expression variations in Down syndrome: impact 
on disease phenotypes. Am J Hum Genet 2007; 81: 475-491.

10. Rachidi M, Lopes C. Mental retardation and associated neurological dysfunctions in Down syndrome: a consequence of dysregulation in critical chromosome 21 genes and associated molecular pathways. Eur J Paediatr Neurol 2008; 12: 168-182.

11. Antonarakis SE, Lyle R, Chrast R, Scott HS. Differential gene expression studies to explore the molecular pathophysiology of Down syndrome. Brain Res Brain Res Rev 2001; 36: 265-274.

12. Mouton-Liger F, Sahun I, Collin T, Lopes Pereira P, Masini D, Thomas S. Developmental molecular and functional cerebellar alterations induced by PCP4/PEP19 overexpression: implications for Down syndrome. Neurobiol Dis 2014; 63: 92-106.

13. Møller RS, Kubart S, Hoeltzenbein M, Heye B, Vogel I, Hansen CP, Menzel C, Ullmann R, Tommerup N, Ropers HH, Tümer Z, Kalscheuer VM. Truncation of the Down syndrome candidate gene DYRK1A in two unrelated patients with microcephaly. Am J Hum Genet 2008; 82: 1165-1170.

14. Mok KY, Jones EL, Hanney M, Harold D, Sims R, Williams J. Polymorphisms in BACE2 may affect the age of onszheimers dementia in Down syndrome. Neurobiol Aging 2014; 35: 1513.

15. Fillat C, Bofill-De Ros X, Santos M, Martín ED, Andreu $\mathrm{N}$, Villanueva $\mathrm{E}$. Identification of key genes involved in Downs syndrome pathogenesis by gene therapy. Int Med Rev Down Syndr 2014; 18: 21-28.

16. Rahmani Z, Blouin JL, Creau-Goldberg N, Watkins PC, Mattei JF, Poissonnier M, Prieur M, Chettouh Z, Nicole A, Aurias A. Critical role of the D21S55 region on chromosome 21 in the pathogenesis of Down syndrome. Proc Natl Acad Sci U S A 1989; 86: 5958-5962.

17. Korenberg J, Kawashima H, Pulst S, Ikeuchi T, Ogasawara N, Yamamoto K. Molecular definition of a region of chromosome 21 that causes features of the Down syndrome phenotype. Am J Human Genet 1990; 47: 236-246.

18. Rachidi M, Lopes C. Mental retardation in Down syndrome: from gene dosage imbalance to molecular and cellular mechanisms. Neurosci Res 2007; 59: 349-369.

19. Montoya J, Soto J, Satizábal J, Sanchez A, García F. Genomic study of the critical region of chromosome 21 associated to Down syndrome. Colom Med 2011; 42: 26-38.

20. Montoya J, Pena A, Satizabal J, Garcia-Vallejo F. In silico systemic analysis of the differential expression of genes located in critical region of Down syndrome in the human brain. Rev Med 2012; 20: 15-26.

21. Allen institute for brain science (http: // www.alleninstitute.org) 2017.

22. Allen brain atlas-data portal (http: //www.brain-map.org) 2017.
23. Documentation Allen human brain atlas (http: // help.brain-map.org/display/humanbrain/Documentation) 2016.

24. Cytoscape 3.1.1 (http: //www.cytoscape.org) 2017.

25. IBM SPSS Statistics 20.0.0 (http: //www.spss.com) 2017.

26. Kahlem P, Sultan M, Herwig R, Steinfath M, Balzereit D, Eppens B. Transcript level alterations reflect gene dosage effects across multiple tissues in a mouse model of Down syndrome. Genome Res 2004; 14: 1258-1267.

27. Lyle R, Gehrig C, Neergaard-Henrichsen C, Deutsch S, Antonarakis S. Gene expression from the aneuploid chromosome in a trisomy mouse model of Down syndrome. Genome Res 2004; 14: 1268-1274.

28. Fuentes J, Pritchard M, Planas A. A new human gene from the Down syndrome critical region encodes a proline-rich protein highly expressed in fetal brain and heart. Hum Mol Genet 1996; 4: 1935-1944.

29. Li CM1, Guo M, Salas M, Schupf N, Silverman W, Zigman WB, Husain S, Warburton D, Thaker H, Tycko B. Cell type-specific over-expression of chromosome 21 genes in fibroblasts and fetal hearts with trisomy 21. BMC Med Genet 2006; 7: 24.

30. Graybiel AM. The basal ganglia: learning new tricks and loving it. Curr Opin Neurobiol 2005; 15: 638-644.

31. Packard MG, Knowlton BJ. Learning and memory functions of the Basal Ganglia. Annu Rev Neurosci 2002; 25: $563-593$.

32. Floresco SB. The nucleus accumbens: an interface between cognition, emotion, and action. Annu Rev Psychol 2014; 66: 20-28.

33. Forbes CE, Grafman J. The role of the human prefrontal cortex in social cognition and moral judgment. Annu Rev Neurosci 2010; 33: 299-324.

34. Squire LR, Wixted JT. The cognitive neuroscience of human memory since H.M. Annu Rev Neurosci 2011; 34: 259-288.

35. Gabrieli JD. Cognitive neuroscience of human memory. Annu Rev Psychol 1998; 49: 87-115.

36. Pujol J, Del Hoyo L, Blanco-Hinojo L, de Sola S, Macia D, Martínez-Vilavella G, Amor M, Deus J, Rodriguez J, Farré M,Dierssen M, de la Torre R. Anomalous brain functional connectivity contributing to poor adaptive behavior in Down syndrome. Cortex 2014; 64: 148-156.

37. Erhardt JA, Legos JJ, Johanson RA, Slemmon JR, Wang $\mathrm{X}$. Expression of PEP-19 inhibits apoptosis in PC12 cells. Neuroreport 2000; 11: 3719-3723.

38. Ichikawa H, Sugimoto T. Peptide 19 in the rat vagal and glossopharyngeal sensory ganglia. Brain Res 2005; 1038: 107-112.

39. Thomas T, Thiery E, Aflalo R, Vayssettes C, Verney C, Berthuy I, Creau N. PCP4 is highly expressed in ectoderm and particularly in neuroectoderm derivatives during mouse embryogenesis. Gene Expression Patterns 2003; 3: 93-97.

40. Mouton-Liger F, Thomas S, Rattenbach R, Magnol L, Larigaldie V, Ledru A, Herault Y, Verney C, Créau N. 
PCP4 (PEP19) overexpression induces premature neuronal differentiation associated with $\mathrm{Ca} 21 /$ Calmodulin-dependent Kinase II-d activation in mouse models of Down Syndrome. J Com Neurol 2011; 519: 2779-2802.

41. Cramer NP, Best TK, Stoffel M, Siarey RJ, Galdzicki Z. GABAB-GIRK2-mediated signaling in Down syndrome. Adv Pharmacol 2010; 58: 397-426.

42. Harashima C, Jacobowitz M, Jassir W, Borke C, Best T, Siarey J, Galdzicki Z. Abnormal expression of the GIRK2 potassium channel in hippocampus, frontal cortex and substantia nigra of Ts65Dn mouse: a model of Down syndrome. J Comparat Neurol 2006; 494: 815-833.

43. Kobayashi $\mathrm{T}$, Washiyama $\mathrm{K}$, Ikeda $\mathrm{K}$. Inhibition of $\mathrm{G}$ protein-activated inwardly rectifying $\mathrm{K}+$ channels by fluoxetine (Prozac). Br J Pharmacol 2003; 138: 1119-1128.

44. Best T, Cramer N, Chakrabarti L, Haydar T, Galdzicki Z. Dysfunctional hippocampal inhibition in the Ts65Dn mouse model of Down syndrome. Exp Neurol 2012; 233: 749-757.

45. Vidal V, Garcia S, Martinez P, Corrales A, Florez J, Rueda N, Sharma A, Martinez-Cué C. Lack of behavioral and cognitive effects of chronic Ethosuximide and Gabapentin treatment in the TS65DN mouse model of Down Syndrome. J Neurosci 2012; 220: 158-168.

46. Park J, Oh Y, Chung K. Two key genes closely implicated with the neuropathological characteristics in Down syndrome: DYRK1A and RCAN1. BMB Rep 2009; 42: 6-15.

47. Song HJ, Park J, Seo S, Kim J, Paik S, Chung K. Down syndrome critical region 2 protein inhibits the transcriptional activity of peroxisome proliferatoractivated receptor $\mathrm{b}$ in HEK293 cells. Biochem Biophys Res Commun 2008; 376: 478-482.

48. Wiechmann S, Czajkowska H, de Graaf K, Grotzinger J, Joost HG, Becker W. Unusual function of the activation loop in the protein kinase DYRK1A. Biochem Biophys Res Commun 2003; 302: 403-408.

49. Lochhead PA, Sibbet G, Morrice N, Cleghon V. Activation-loop autophosphorylation is mediated by a novel transitional intermediate form of DYRKs. Cell 2005; 121: 925-936.

50. Guimera J, Casas C, Estivill X, Pritchard M. Human minibrain homologue (MNBH/DYRK1): characterization, alternative splicing, differential tissue expression, and overexpression in Down syndrome. Genomics 1999; 57 : 407-418.

51. Shindoh N, Kudoh J, Maeda H, Yamaki A, Minoshima S, Shimizu Y, Shimizu N. Cloning of a human homolog of the Drosophila minibrain/rat Dyrk gene from the Down syndrome critical region of chromosome 21. Biochem Biophys Res Commun 1996; 225: 92-99.

52. Song WJ, Sternberg LR, Kasten-Sportes C, Keuren ML, Chung SH, Slack AC, Miller DE, Glover TW, Chiang PW, Lou L, Kurnit DM. Isolation of human and murine homologues of the Drosophila minibrain gene: human homologue maps to 21q22.2 in the Down syndrome critical region. Genomics 1996; 38: 331-339.

53. Lockstone HE, Harris LW, Swatton JE, Wayland MT, Holland AJ, Bahn S. Gene expression profiling in the adult Down syndrome brain. Genomics 2007; 90: 647-660.

54. Ruffner H, Bauer A, Bouwmeester T. Human proteinprotein interaction networks and the value for drug discovery. Drug Discov Today 2007; 12: 709-716.

55. Sporns O, Tononi G, Edelman GM. Connectivity and complexity: the relationship between neuroanatomy and brain dynamics. Neural Netw 2000; 13: 909-922.

56. Mao R, Wang X, Spitznagel E, Frelin L, Ting J, Ding H, Kim¥ J, Ruczinski I, Downey T, Pevsner J. Primary and secondary transcriptional effects in the developing human Down syndrome brain and heart. Genome Biol 2005; 6: 107.

\section{*Correspondence to}

Dianora Fajardo

Department of Biochemistry

Institución Universitaria Escuela Nacional del Deporte

Colombia 